\title{
Millikelvin cooling of an optically trapped microsphere in vacuum
}

\author{
Tongcang Li, Simon Kheifets and Mark G. Raizen ${ }^{\star}$
}

Cooling of micromechanical resonators towards the quantum mechanical ground state in their centre-of-mass motion has advanced rapidly in recent years ${ }^{1-8}$. This work is an important step towards the creation of 'Schrödinger cats', quantum superpositions of macroscopic observables, and the study of their destruction by decoherence. Here we report optical trapping of glass microspheres in vacuum with high oscillation frequencies, and cooling of the centre-of-mass motion from room temperature to a minimum temperature of about $1.5 \mathrm{mK}$. This new system eliminates the physical contact inherent to clamped cantilevers, and can allow ground-state cooling from room temperature ${ }^{9-15}$. More importantly, the optical trap can be switched off, allowing a microsphere to undergo free-fall in vacuum after cooling ${ }^{15}$. This is ideal for studying the gravitational state reduction ${ }^{16-19}$, a manifestation of the apparent conflict between general relativity and quantum mechanics ${ }^{16,20}$. A cooled optically trapped object in vacuum can also be used to search for non-Newtonian gravity forces at small scales ${ }^{21}$, measure the impact of a single air molecule ${ }^{14}$ and even produce Schrödinger cats of living organisms ${ }^{9}$.

Previous experiments demonstrated optical levitation of a $20-\mu \mathrm{m}$-diameter sphere in vacuum with a trapping frequency of about $20 \mathrm{~Hz}$, as well as feedback control of a trapped sphere that was used to increase the trapping frequency to several hundred hertz and stabilize its position to within a fraction of one micrometre ${ }^{22,23}$. However, the resolution of its detection system ${ }^{23}$ was not sufficient to enable feedback cooling. According to the equipartition theorem, the root-mean-square (r.m.s.) amplitude of a trapped microsphere at thermal equilibrium is $x_{\text {r.m.s. }}=\sqrt{k_{\mathrm{B}} T_{0} /\left(m \omega^{2}\right)}$, where $k_{\mathrm{B}}$ is the Boltzmann constant, $T_{0}$ is the air temperature, $m$ is the mass of the microsphere and $\omega$ is the angular trapping frequency. For a $20-\mu \mathrm{m}$-diameter sphere trapped at one-hundred hertz, the r.m.s. amplitude is about $0.04 \mu \mathrm{m}$ at $300 \mathrm{~K}$, and will be much smaller at lower temperature. It is also important that the trapping frequency be much higher than the frequencies of seismic vibration to achieve significant cooling.

We use a dual-beam optical tweezer to trap a 3.0- $\mu$ mdiameter $\mathrm{SiO}_{2}$ sphere in vacuum with much higher oscillation frequencies (about $10 \mathrm{kHz}$ ) to minimize the effects of instrumental vibration. We also demonstrate a detection system to monitor the motion of a trapped microsphere with a sensitivity of about $39 \mathrm{fm} \mathrm{Hz} z^{-1 / 2}$ over a wide frequency range. Using active feedback, we simultaneously cool the three centre-of-mass vibration modes of a microsphere from room temperature to millikelvin temperatures with a minimum mode temperature of $1.5 \mathrm{mK}$, which corresponds to the reduction of the r.m.s. amplitude of the microsphere from $6.7 \mathrm{~nm}$ to $15 \mathrm{pm}$ for that mode.

A simplified scheme of our optical trap and feedback set-up is shown in Fig. 1. The optical trap is similar to a trap used to measure the instantaneous velocity of a Brownian particle in $\operatorname{air}^{24}$. It is created inside a vacuum chamber by two counter-propagating laser beams focused to the same point by two identical aspheric lenses with a focal length of $3.1 \mathrm{~mm}$ and a numerical aperture of 0.68 . The wavelength of both trapping beams is $1,064 \mathrm{~nm}$. They are orthogonally polarized, and are shifted in frequency to avoid interference. The beams are slightly elliptical and approximately form a harmonic trap with three fundamental vibration modes along the horizontal, vertical and axial directions, denoted $X, Y$ and $Z$ in Fig. 1a. The motion of a trapped bead causes deflection of both trapping beams. We monitor the position of the bead by measuring the deflection of one of the trapping beams with ultrahigh spatial and temporal resolution in all three dimensions (Supplementary Fig. S1).

Using the position signal, we can calculate the instantaneous velocity of the bead, and implement feedback cooling by applying a force with a direction opposing the velocity (Fig. 1b). The feedback is generated by scattering forces from three orthogonal $532 \mathrm{~nm}$ laser beams along the axes as shown in Fig. 1a. The average intensity of the cooling beams is about $1 \%$ of the trapping beams. The optical power of each cooling beam is controlled by an acousto-optic modulator (AOM). Each beam is modulated with a time-varying signal proportional to the instantaneous velocity of the bead, added to an offset. The proportional component generates the required cooling force, and the offset slightly shifts the trap centre.

An optically trapped microsphere in non-perfect vacuum will exhibit Brownian motion due to collisions between the microsphere and residual air molecules. When the microsphere is at thermal equilibrium, the power spectrum of centre-of-mass motion along each of the three fundamental mode axes is ${ }^{5,25}$ :

$$
S_{j}(\Omega)=\frac{2 k_{\mathrm{B}} T_{0}}{m} \frac{\Gamma_{0}}{\left(\omega_{j}^{2}-\Omega^{2}\right)^{2}+\Omega^{2} \Gamma_{0}^{2}}
$$

where $\Omega / 2 \pi$ is the observation frequency, $\Gamma_{0}$ is the viscous damping factor due to the air and $\omega_{j}(j=1,2,3)$ are the resonant frequencies of the optical trap along the $X, Y$ and $Z$ axes.

The viscous damping factor due to air can be calculated by kinetic theory. Assuming the reflection of air molecules from the surface of a microsphere is diffusive, and the molecules thermalize with the surface during collisions, we have ${ }^{26}$ :

$$
\Gamma_{0}=\frac{6 \pi \eta r}{m} \frac{0.619}{0.619+\mathrm{Kn}}\left(1+c_{K}\right)
$$

where $\eta$ is the viscosity coefficient of the air, $r$ is the radius of the microsphere and $\mathrm{Kn}=s / r$ is the Knudsen number. Here, $s$ is the mean free path of the air molecules. $c_{K}=$ $(0.31 \mathrm{Kn}) /\left(0.785+1.152 \mathrm{Kn}+\mathrm{Kn}^{2}\right)$ is a small positive function of 


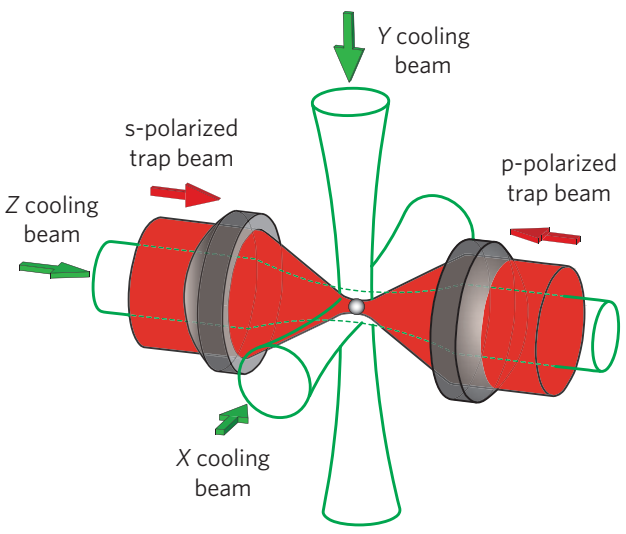

b

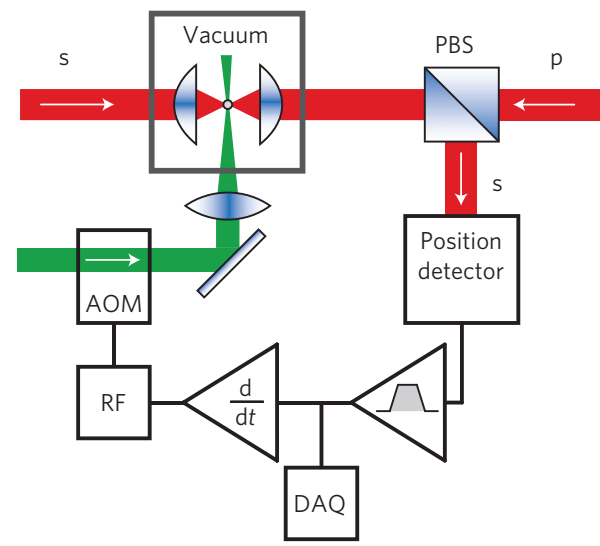

Figure 1 | Schematic of feedback cooling of an optically trapped microsphere. a, Simplified schematic showing a glass microsphere trapped at the focus of a counter-propagating dual-beam optical tweezer, with three laser beams along the axes for cooling. The wavelengths of the trapping beams and the cooling beams are $1064 \mathrm{~nm}$ and $532 \mathrm{~nm}$, respectively. b, Diagram of the feedback mechanism for the $X$ axis. The position of a trapped microsphere is monitored by a home-built detection system. The position signal is sent through a band-pass filter (typically $100 \mathrm{~Hz}-300 \mathrm{kHz}$ ) and a derivative circuit (d/dt) to provide a signal proportional to velocity. This velocity signal is used to control the output power of a radiofrequency (RF) AOM driver that modulates the power of the $X$ cooling beam. The data are digitized and stored on a computer by a data acquisition card (DAQ). PBS, polarizing beam splitter.

$\mathrm{Kn}$. At low pressures where $\mathrm{Kn} \gg 1$, the viscous damping factor is proportional to the pressure.

The behaviour of the system with three-dimensional feedback cooling is straightforward to understand if we assume that there is no coupling between feedback forces and velocities in different directions. In this case, the feedback force in each direction adds an effective cold damping factor $\Gamma_{j}^{\mathrm{fb}}$, and the total damping becomes $\Gamma_{j}{ }^{\text {tot }}=\Gamma_{0}+\Gamma_{j}{ }^{\mathrm{fb}}$. The power spectrum of the motion of a trapped microsphere with feedback cooling can be described by equation (1) with $T_{0}$ and $\Gamma_{0}$ replaced by $T_{j}^{\mathrm{fb}}$ and $\Gamma_{j}^{\text {tot }}$, where $T_{j}^{\mathrm{fb}}=T_{0} \Gamma_{0} / \Gamma_{j}{ }^{\text {tot }}$ is the temperature of the motion with feedback cooling ${ }^{5}$. The motion can be cooled significantly by applying feedback damping $\Gamma_{j}^{\mathrm{fb}} \gg \Gamma_{0}$. The lowest temperature will be limited by the noise in the detection system and feedback circuits, as well as coupling between different directions.

Figure 2 shows the linewidths, $\Gamma_{0} / 2 \pi$, of the oscillation of a trapped 3- $\mu \mathrm{m}$ microsphere at different pressures without feedback cooling. The powers of the two trapping beams are $120 \mathrm{~mW}$ and $100 \mathrm{~mW}$, respectively. The linewidths are obtained by fitting the measured power spectra with equation (1). The measured

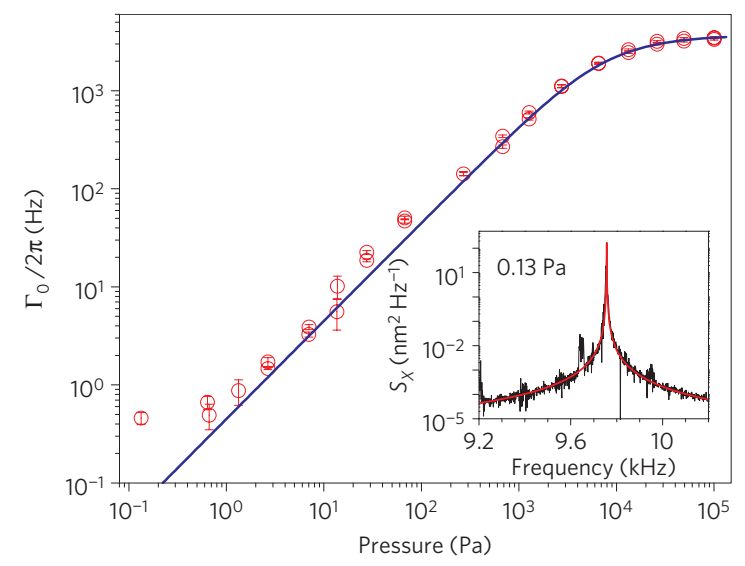

Figure 2 | Measured linewidths of the oscillation of an optically trapped 3-um-diameter microsphere at different pressures. The blue curve is the prediction of a kinetic theory (equation (2)). The inset is the measured power spectrum at $0.13 \mathrm{~Pa}$. By fitting the spectrum with equation (1) (red curve), we obtain $\omega_{1}=2 \pi(9756.4 \pm 0.3) \mathrm{Hz}$ and $\Gamma_{0}=2 \pi(0.46 \pm 0.06) \mathrm{Hz}$ for this example. The same method is used to obtain linewidths for other pressures. Error bars indicate the standard errors in the fitting of the power spectra.

linewidths agree very well with the prediction of kinetic theory (equation (2)) from $10^{5} \mathrm{~Pa}$ down to $1 \mathrm{~Pa}$. At pressures below $1 \mathrm{~Pa}$, the measured linewidths are larger than the theoretical prediction. This linewidth broadening is due to power fluctuations of the trapping laser. The inset of Fig. 2 shows a power spectrum at $0.13 \mathrm{~Pa}$. The trapping frequency $\omega_{1} / 2 \pi$ is $9,756.4 \pm 0.3 \mathrm{~Hz}$, and the linewidth is $0.46 \pm 0.06 \mathrm{~Hz}$, giving a quality factor $\left(Q_{j}=\omega_{j} / \Gamma_{0}\right)$ of $2.1 \times 10^{4}$. This implies the power fluctuation of the trapping laser is smaller than $0.01 \%$ during the measurement. An optically trapped microsphere provides a method to directly convert laser power to a frequency signal, which can be measured precisely. Stabilization of laser power to a trapped bead can find applications in laser physics, and can enable a more precise measurement of the $Q$ for a second trapped bead in vacuum.

Figure 3 shows experimental results of feedback cooling. Before feedback is turned on, the resonant frequencies $\omega_{j} / 2 \pi$ are $8,066 \pm 5 \mathrm{~Hz}, 9,095 \pm 4 \mathrm{~Hz}$ and $2,072 \pm 6 \mathrm{~Hz}$ for the fundamental modes at $637 \mathrm{~Pa}$ along the $X, Y$ and $Z$ axes, respectively. At this pressure, the peaks in the power spectrum due to the three fundamental modes are distinguishable, and heating effects due to the laser are negligible. We can therefore use the measured power spectra at $637 \mathrm{~Pa}$ to calibrate the position detectors for the fundamental modes at room temperature. After we turn on feedback cooling, the temperature of the $Y$ mode changes from 297 to $24 \mathrm{~K}$ at $637 \mathrm{~Pa}$. Then we reduce the air pressure while keeping the feedback gain almost constant; thus, the heating rate due to collisions from air molecules decreases, while the cooling rate remains constant. As a result, the temperature of the motion drops. At $5.2 \mathrm{mPa}$, the mode temperatures are $150 \pm 8 \mathrm{mK}, 1.5 \pm 0.2 \mathrm{mK}$ and $68 \pm 5 \mathrm{mK}$ for the $X, Y$ and $Z$ modes. The mean thermal occupancy $\langle n\rangle=k_{\mathrm{B}} T_{j}^{\mathrm{fb}} /\left(\hbar \omega_{j}\right)$ of the $Y$ mode is reduced from about $6.8 \times 10^{8}$ at $297 \mathrm{~K}$ to about 3,400 at $1.5 \mathrm{mK}$. Here, $\hbar$ is the reduced Planck constant. Figure $3 \mathrm{~d}$ shows the temperature of the three fundamental modes as a function of pressure. At low pressure and when the feedback gain is constant, the mode temperature should be proportional to the pressure, which is shown as a straight line with slope 1 in the figure. The temperature of the $Y$ mode agrees with this prediction very well at pressures above $1 \mathrm{~Pa}$.

At our lowest temperatures, the power spectra are still much larger than the noise level, and the minimum temperature is achieved at pressures above the minimum pressure we can 

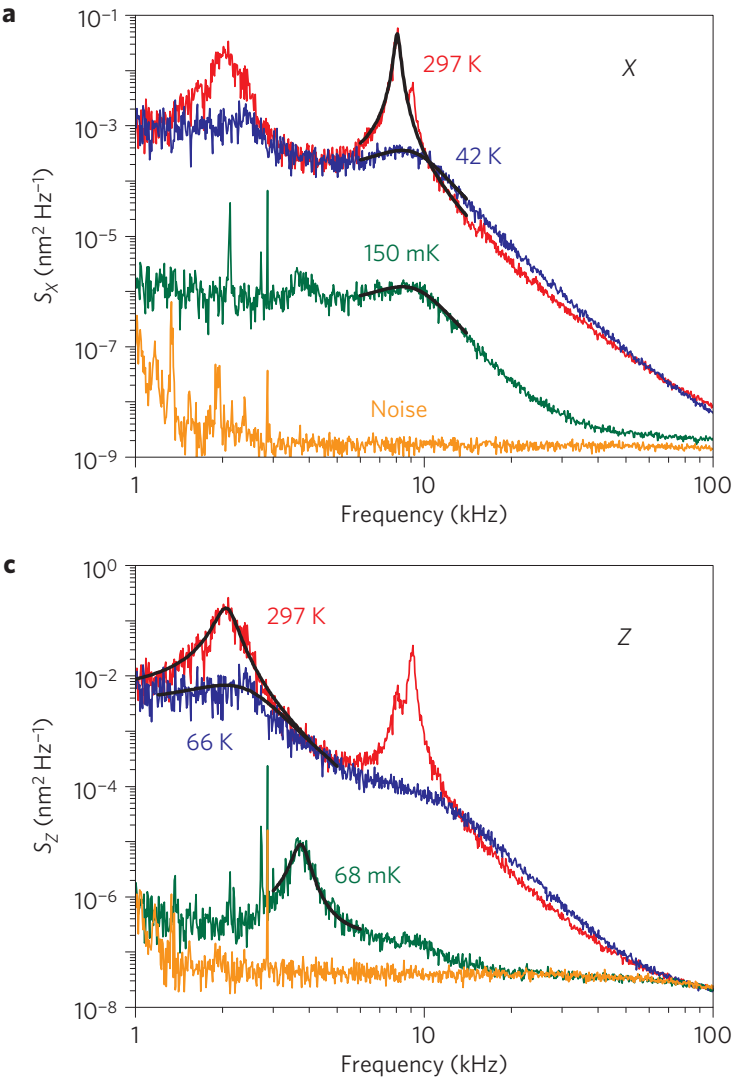
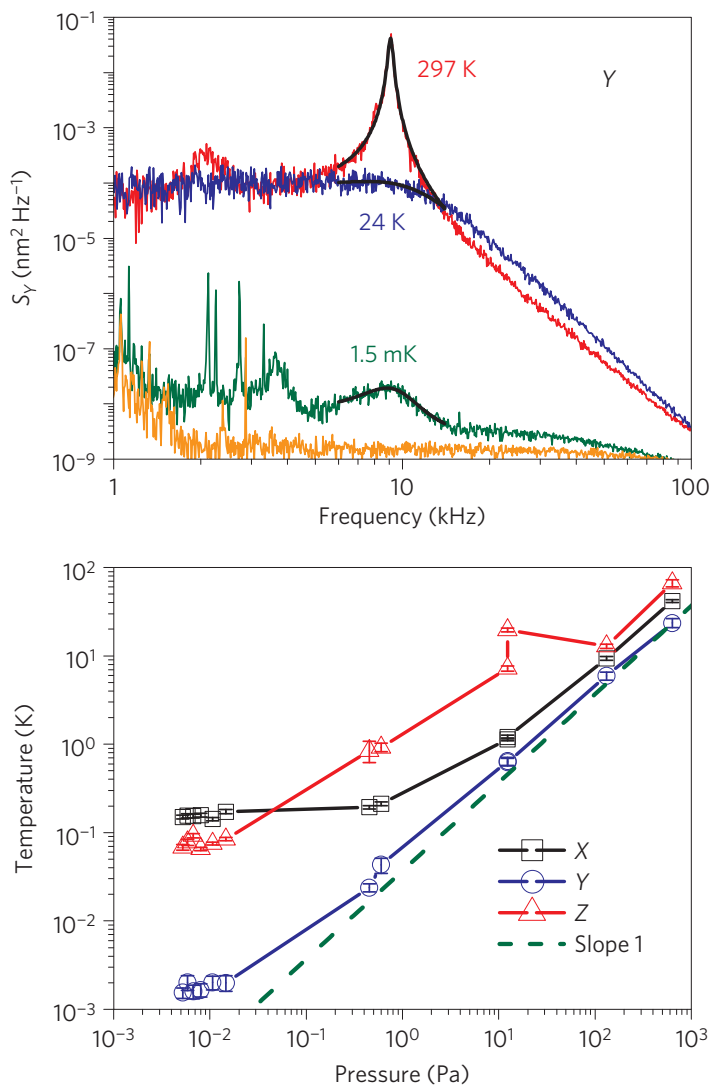

Figure 3 | Experimental results of feedback cooling. a-c, The power spectra of a trapped 3- $\mu$ m-diameter microsphere along the $X(\mathbf{a}), Y(\mathbf{b})$ and $Z$ (c) axes as it is cooled. Red curves are intrinsic spectra at $637 \mathrm{~Pa}$ without feedback cooling, blue curves are spectra at 637 Pa with feedback cooling, green curves are spectra at $5.2 \mathrm{mPa}$ with feedback cooling and orange curves are the noise signals when there is no particle in the optical trap. The black curves are fits of a thermal model (see text). We obtain mode temperatures from these fits. $\mathbf{d}$, Temperatures of the three fundamental oscillation modes along $X$ (black squares), $Y$ (blue circles) and $Z$ (red triangles) axes as a function of the air pressure. Error bars indicate the standard errors in the fitting of the power spectra. The dashed line is a straight line with slope 1 for comparison.

obtain; thus, the electronic noise (in detection and feedback circuits) and the pressure are not the limiting factors of the present experiment. The dominant limiting factor is most likely residual coupling between intensity and direction of the cooling beams. When we change the intensity of a cooling beam by an $\mathrm{AOM}$, the direction and profile of the beam is also changed slightly. This causes heating of the motion of a microsphere perpendicular to the beam while cooling it parallel. This problem should be solved by replacing the AOMs with electro-optic modulators. The final temperature limited by the present detection system will be about $0.1 \mathrm{mK}$. Right now, the laser beam is attenuated before entering the detectors because the laser power is larger than the damage threshold of the detectors. If we can use all of the signal contained in the laser beam for feedback cooling, the final temperature can be smaller than $0.01 \mathrm{mK}$, corresponding to a thermal occupation number of the order of 10 or less.

With feedback cooling, we have trapped a microsphere for more than one hour at pressure below $1 \mathrm{mPa}$. This lifetime should be long enough to carry out cavity cooling ${ }^{9,10,14}$. Our three-dimensional cooling enables future work on quantum superposition and entanglement of the motion between different directions. We have also been able to charge the microsphere by high-voltage electrical breakdown of nearby air. After charging, we use electrostatic forces to implement feedback cooling, and have been able to cool the motion of a trapped microsphere to about $10 \mathrm{~K}$. This provides a method for combining an optical trap and an ion trap ${ }^{27}$ at one place, which will help to trap and study particles at ultrahigh vacuum.
Our result is an important step towards quantum ground-state cooling of a trapped macroscopic object in vacuum by either cavity cooling $9,10,14$ or feedback cooling with an improved detection and feedback scheme $e^{28,29}$. For cavity cooling of a trapped object in vacuum, it is also important to use feedback cooling to pre-cool and stabilize the object, to have enough time to tune the cavity-cooling laser to the correct frequency for efficient cooling. After cooling and creation of a superposition state in momentum, the optical trap can be switched off to let a microsphere undergo free-fall in vacuum ${ }^{15}$. The wavefunction will expand during free-fall and become a superposition state in space. The finite lifetime of a superposition due to gravity-induced state reduction is predicted to be of the order of $\hbar r /\left(\mathrm{Gm}^{2}\right)$ when the superposition is composed of states separated by a distance larger than the size of the microsphere ${ }^{16,19}$, where $G$ is Newton's gravitational constant. The predicted lifetime is about $3 \mathrm{~ms}$ for a 3 - $\mu \mathrm{m}$-diameter microsphere, which is shorter than the environmental decoherence time in good vacuum and thus measurable.

Received 3 January 2011; accepted 14 February 2011; published online 20 March 2011

\section{References}

1. Metzger, C. H. \& Karrai, K. Cavity cooling of a microlever. Nature 432, 1002-1005 (2004)

2. Naik, A. et al. Cooling a nanomechanical resonator with quantum back-action. Nature 443, 193-196 (2006).

3. Gigan, S. et al. Self-cooling of a micromirror by radiation pressure. Nature 444, 67-70 (2006). 
4. Arcizet, O., Cohadon, P-F., Briant, T., Pinard, M. \& Heidmann, A. Radiation-pressure cooling and optomechanical instability of a micromirror. Nature 444, 71-74 (2006).

5. Kleckner, D. \& Bouwmeester, D. Sub-kelvin optical cooling of a micromechanical resonator. Nature 444, 75-78 (2006).

6. Schliesser, A., Del'Haye, P., Nooshi, N., Vahala, K. J. \& Kippenberg, T. J. Radiation pressure cooling of a micromechanical oscillator using dynamical backaction. Phys. Rev. Lett. 97, 243905 (2006).

7. Thompson, J. D. et al. Strong dispersive coupling of a high-finesse cavity to a micromechanical membrane. Nature 452, 72-75 (2008).

8. O'Connell, A. D. et al. Quantum ground state and single-phonon control of a mechanical resonator. Nature 464, 697-703 (2010).

9. Romero-Isart, O., Juan, M. L., Quidant, R. \& Cirac, J. I. Toward quantum superposition of living organisms. New J. Phys. 12, 033015 (2010).

10. Chang, D. E. et al. Cavity opto-mechanics using an optically levitated nanosphere. Proc. Natl Acad. Sci. USA 107, 1005-1010 (2010).

11. Singh, S., Phelps, G. A., Goldbaum, D. S., Wright, E. M. \& Meystre, P. All-optical optomechanics: An optical spring mirror. Phys. Rev. Lett. 105, 213602 (2010).

12. Barker, P. F. Doppler cooling a microsphere. Phys. Rev. Lett. 105, 073002 (2010)

13. Schulze, R. J., Genes, C. \& Ritsch, H. Optomechanical approach to cooling of small polarizable particles in a strongly pumped ring cavity. Phys. Rev. A 81, 063820 (2010).

14. Yin, Z. Q., Li, T. \& Feng, M. Three-dimensional cooling and detection of a nanosphere with a single cavity. Phys. Rev. A 83, 013816 (2011).

15. Romero-Isart, O. et al. Optically levitating dielectrics in the quantum regime: Theory and protocols. Phys. Rev. A 83, 013803 (2011).

16. Penrose, R. On gravity's role in quantum state reduction. Gen. Rel. Grav. 28, 581-600 (1996).

17. Diósi, L. Models for universal reduction of macroscopic quantum fluctuations Phys. Rev. A 40, 1165-1174 (1989).

18. Ghirardi, G. C., Rimini, A. \& Weber, T. Unified dynamics for microscopic and macroscopic systems. Phys. Rev. D 34, 470-491 (1986).

19. van Wezel, J., Oosterkamp, T. \& Zaanen, J. Towards an experimental test of gravity-induced quantum state reduction. Phil. Mag. 88, 1005-1026 (2008).

20. Hawking, S. W. \& Israel, W. (eds) General Relativity; an Einstein Centenary Survey (Cambridge Univ. Press, 1979).
21. Geraci, A. A., Papp, S. B. \& Kitching, J. Short-range force detection using optically cooled levitated microspheres. Phys. Rev. Lett. 105, 101101 (2010).

22. Ashkin, A. \& Dziedzic, J. M. Optical levitation in high vacuum. Appl. Phys. Lett. 28, 333-335 (1976)

23. Ashkin, A. \& Dziedzic, J. M. Feedback stabilization of optically levitated particles. Appl. Phys. Lett. 30, 202-204 (1977).

24. Li, T., Kheifets, S., Medellin, D. \& Raizen, M. G. Measurement of the instantaneous velocity of a Brownian particle. Science 328, 1673-1675 (2010).

25. Berg-Sørensen, K. \& Flyvbjerg, H. Power spectrum analysis for optical tweezers. Rev. Sci. Instrum. 75, 594-612 (2004).

26. Beresnev, S. A., Chernyak, V. G. \& Fomyagin, G. A. Motion of a spherical particle in a rarefied gas. Part 2. Drag and thermal polarization. J. Fluid Mech. 219, 405-421 (1990).

27. Hinkle, L. D. \& Kendall, B. R. F. Pressure-dependent damping of a particle levitated in vacuum. J. Vac. Sci. Technol. A 8, 2802-2805 (1990).

28. Mancini, S., Vitali, D. \& Tombesi, P. Optomechanical cooling of a macroscopic oscillator by homodyne feedback. Phys. Rev. Lett. 80, 688-691 (1998).

29. Genes, C., Vitali, D., Tombesi, P., Gigan, S. \& Aspelmeyer, M. Ground-state cooling of a micromechanical oscillator: Comparing cold damping and cavity-assisted cooling schemes. Phys. Rev. A 77, 033804 (2008).

\section{Acknowledgements}

We thank O. Romero-Isart for discussion of using a cooled microsphere to test objective reduction of the wavefunction models. We also thank D. Medellin and Z. Q. Yin for helpful discussions. M.G.R. acknowledges support from the Sid W. Richardson Foundation and the R. A. Welch Foundation, grant number F-1258. T.L. acknowledges support from the Lawrence C. Biedenharn Endowment for Excellence.

\section{Author contributions}

T.L. and M.G.R. designed the project. S.K. and T.L. carried out the experiment. All authors wrote the paper.

\section{Additional information}

The authors declare no competing financial interests. Supplementary information accompanies this paper on www.nature.com/naturephysics. Reprints and permissions information is available online at http://npg.nature.com/reprintsandpermissions. Correspondence and requests for materials should be addressed to M.G.R. 